\title{
What is trogocytosis and what is its purpose?
}

\section{To the editor:}

Several reports have documented that lymphocytes can extract surface molecules through the 'immunological synapse' from the antigen-presenting cells to which they are conjugated ${ }^{1}$. This phenomenon, which we have called 'trogocytosis' ${ }^{1}$ (from the ancient Greek trogo, meaning 'gnaw'), involves the transfer of plasma membrane fragments from the presenting cell to the lymphocyte. Trogocytosis has been documented in T, B and natural killer cells both in vitro and in vivo. Thus, this process may be important in the induction and regulation of immune responses, and possibly in the control of other cellular systems ${ }^{1}$.

Trogocytosis corresponds to an active transfer phenomenon that is triggered specifically by antigen receptor signaling. It occurs within minutes of conjugate formation between two live cells and therefore cannot be the result of phagocytosis of apoptotic bodies ${ }^{2-4}$. Trogocytosis by cytotoxic T lymphocytes might contribute to the selection of high-affinity T cells through the removal of antigenic complexes from the presenting dendritic cells ${ }^{5}$ and to the elimination of cytotoxic T lymphocytes through 'fratricide' after the acquisition of major histocompatibility complex molecules loaded with antigenic peptide recognized by their own T cell receptor ${ }^{2}$. Such 'fratricide' may not be physiological, however, as it only occurs when an extremely high concentration of major histocompatibility complex class I-restricted antigenic peptide is added externally to the target cell ${ }^{2}$.

We postulate that trogocytosis could have a broader function in the biology of lymphocytes. During trogocytosis, lymphocytes inherit many different molecules from conjugating cells. Some of these molecules, which are not transcribed by lymphocytes, may directly or indirectly influence the phenotype and function of the lymphocytes. From an evolutionary perspective, trogocytosis may have developed initially as a symbiotic arrangement: leukocytes may be 'feeding' off other cell types in return for undertaking the defense of the organism against pathogens. Because lipids are the most energetically demanding components to generate, fragments of plasma membrane acquired by lymphocytes could contribute substantially to their metabolic balance, thereby increasing their capacity to proliferate.

Trogocytosis could be a vector for intercellular communication. Molecules acquired during trogocytosis that might alter the lymphocytes' activity include regulatory molecules on either side of the plasma membrane, such as extracellular (hormone receptors and costimulatory molecules) or intracellular (signal transduction components and transcription factors) components. The acquired molecules (for example, 'homing' receptors) could conceivably direct certain lymphocyte functions, such as migration to the appropriate injured tissues.

Lymphocyte activation may also be facilitated through trogocytosis by prolongation of the engagement of antigen receptors after separation of the antigenpresenting cell-lymphocyte conjugate. This process may occur either in endocytic vesicles or in folds of the plasma membrane if the formation of a membrane bridge has occurred, as has been shown ${ }^{3}$. Such a phenomenon would also allow for the integration and accrual of fractional stimulatory signals through successive encounters with antigen-presenting cells.

Finally, trogocytosis may also have negative consequences. Active lymphocytes naturally spend time in close proximity to pathogens. Because of their mobility, lymphocytes could contribute to the spread of pathogens within the host either through direct capture of the pathogen or its genome $^{6}$ (for example, prions or human immunodeficiency virus) or of cell surface viral receptors from other cells ${ }^{7}$.

We believe trogocytosis may have first appeared in very primitive organisms as a way for specialized cell types to feed off other cells. Subsequently, direct transfer of regulatory molecules may have contributed to the establishment of intercellular communications. Of course, lymphocyte activation through trogocytosis would have appeared much later in evolution. The efficient use of available resources through trogocytosis, as proposed here, indicates that evolution may favor this process. Thus, its relative physiological relevance, which is still unclear, is worth investigating.

\section{Etienne Joly $\&$ Denis Hudrisier}

Institut Claude de Préval, U563 INSERM, CHU Purpan, Toulouse, France.

e-mail:atn@cict.fr

1. Hudrisier, D. \& Joly, E. Plasma membrane nibbling: all lymphocytes do it, but why? ELSO Gaz. 9, 1-5 (2002).

2. Huang, J.F. et al. TCR-mediated internalization of peptide-MHC complexes acquired by T cells. Science 286, 952-954 (1999).

3. Stinchcombe, J.C., Bossi, G., Booth, S. \& Griffiths, G.M. The immunological synapse of CTL contains a secretory domain and membrane bridges. Immunity 15, 751-761 (2001).

4. Hudrisier, D., Riond, J., Mazarguil, H., Gairin, J.E. \& Joly, E. Cutting edge: CTLs rapidly capture membrane fragments from target cells in a TCR signaling-dependent manner. J. Immunol. 166, 3645-3649 (2001).

5. Kedl, R.M., Schaefer, B.C., Kappler, J.W. \& Marrack, P. T cells down-modulate peptide-MHC complexes on APCs in vivo. Nat. Immunol. 3, 27-32 (2002).

6. Igakura, T. et al. Spread of HTLV-I between lymphocytes by virus-induced polarization of the cytoskeleton. Science 299, 1713-1716 (2003).

7. Tabiasco, J. et al. Acquisition of viral receptor by NK cells through immunological synapse. J. Immunol. 170, 5993-5998 (2003). 